\title{
Teleotemporalidad: un nivel de estudio adicional para la organización derivado del modelo de Julius Thomas Fraser
}

Teleotemporality: An Additional Study Level for the Organization Derived from the Julius Thomas Fraser Model

Teleotemporalidade: um nível de estudo adicional para a organização derivado do modelo de Julius Thomas Fraser

\section{Claudio Mera Ruiz*}

Fecha de recibido: 10 de mayo de 2015

Fecha de aprobado: 16 de octubre de 2015

Doi: dx.doi.org/10.12804/rev.univ.empresa.30.2016.08

Para citar este artículo: Mera Ruiz, C. (2016). Teleotemporalidad: un nivel de estudio adicional para la organización derivado del modelo de Julius Thomas Fraser. Universidad \& Empresa, 18(30), 159-177. Doi: dx. doi. org/10.12804/rev.univ.empresa.30.2016.08

\section{RESUMEN}

Este artículo propone la teleotemporalidad como un nuevo espacio de análisis de la organización derivado de la consideración del modelo de Julius Thomas Fraser. Para desarrollar la propuesta se sintetiza el trabajo de Fraser, que está compuesto por seis niveles de estudio de la temporalidad: 1) atemporalidad, que toca los fenómenos electromagnéticos; 2) prototemporalidad, que explica el comportamiento molecular; 3) eotemporalidad, que se ocupa de la mecánica clásica; 4) biotemporalidad, que atañe al comportamiento de los seres vivos; 5) nootemporalidad, que explica la experiencia humana, y 6) sociotemporalidad, sobre los

\footnotetext{
Doctor en Ciencias de la Administración, de la Universidad Nacional Autónoma de México (UNAM), magíster en Administración y administrador de empresas, de la Universidad Nacional de Colombia. Correo electrónico: claudio.mera1@gmail.com
} 
fenómenos sociales del tiempo. Por último, se explica la teleotemporalidad como un nuevo espacio de análisis útil para aproximarse a la comprensión de la temporalidad organizacional. Palabras clave: análisis organizacional, Julius Thomas Fraser, organizaciones, teleotemporalidad, temporalidad.

\section{ABSTRACT}

This article proposes teleotemporality as a new space for organizational analysis resulting from consideration of the Julius Thomas Fraser model. To develop the proposal, Fraser"s work is summarized. The work of this author is composed of six study levels of temporality: 1) atemporality, concerning electro-magnetic phenomena; 2) protemporality, which explains molecular behavior; 3) eotemporality, which deals with classical mechanics; 4) biotemporality, concerning the behavior of living beings; 5) nootemporality, which explains human experience, and 6) sociotemporality, about the social phenomena of time. Finally, teleotemporality is explained as a new analysis space useful to understand the organizational temporality.

Keywords: Organizational analysis, Julius Thomas Fraser, organizations, teleotemporality, temporality.

\section{RESUMO}

Este artigo propõe a teleotemporalidade como um novo espaço de análise da organização derivada da consideração do modelo de Julius Thomas Fraser. Para desenvolver a proposta se sintetiza o trabalho de Fraser que está composto por seis níveis de estudo da temporalidade: 1) atemporalidade, que toca os fenómenos eletromagnéticos; 2) prototemporalidade, que explica o comportamento molecular; 3) eotemporalidade, que se ocupa da mecânica clássica; 4) biotemporalidade, que diz respeito ao comportamento dos seres vivos; 5) nootemporalidade, que explica a experiência humana, e 6) sociotemporalidade como um novo espaço de análise útil para se aproximar à compreensão da temporalidade organizacional.

Palavras-chave: análise organizacional, Julius Thomas Fraser, organizações, teleotemporalidade, temporalidade. 


\section{INTRODUCCIÓN}

Este artículo propone la teleotemporalidad como un nivel de análisis adicional en el modelo de estudio del tiempo de Julius Thomas Fraser $(1975 ; 1987)$. Esto se hace con el fin de aportar a la actual comprensión de los estudios organizacionales acerca de los fenómenos temporales propios de los grupos de personas orientados por objetivos.

El modelo desarrollado por Fraser explica el tiempo como un fenómeno ubicuo que presenta diferentes comportamientos, de acuerdo con el análisis que se esté aplicando. En la medida en la que el objeto de estudio se hace más amplio, se van involucrando conceptos y disciplinas científicas adicionales para explicar los fenómenos temporales.

Fraser propone seis capas de la realidad —o niveles de análisis - que se sintetizan en el presente artículo en orden creciente de complejidad. La teleotemporalidad, que proponemos en este texto, se insertaría entre el quinto nivel (correspondiente a la nootemporalidad, que estudia el tiempo de los individuos) y el sexto (sociotemporalidad, que estudia el tiempo de las sociedades) del modelo de este autor.

\section{EL MODELO DE ESTUDIO DE LA TEMPORALIDAD DE FRASER}

La mirada interdisciplinar de Fraser constituye una propuesta comprensiva de la temporalidad como un fenómeno esencial de la realidad (Montoya Restrepo \& Montoya Restrepo, 2009), en oposición a otras explicaciones desde una sola disciplina, como el éxito de ventas de Stephen Hawking (1995). El modelo sostiene que el tiempo debe explicarse desde una perspectiva interdisciplinar y que es necesario analizar el universo como si estuviera constituido por capas. $\mathrm{Si}$ bien cada una de ellas exhibe características independientes, deben considerarse en una relación de orden, pues el desarrollo de una capa requiere la existencia de la anterior (Fraser, 1975).

Cada una de las seis capas del modelo - o umwelts, como son llamadas en la obra original - posee ciertas características únicas y representa un nivel de estudio y naturaleza de los objetos de atención diferente (figura 1). En efecto, las capas de realidad tienen características particulares y explican fenómenos diferenciados en su dimensión temporal (Fraser, 1987). 
Figura 1. Seis capas de la temporalidad en la obra de Fraser

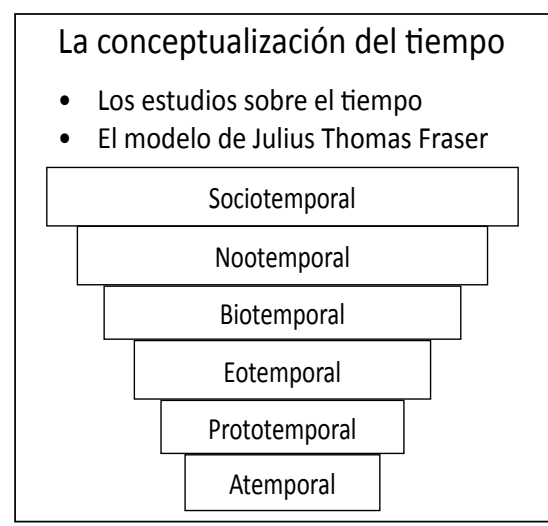

Fuente: elaboración propia con base en Fraser (1975; 1987).

Como ya se ha insinuado, las capas consideradas y las características temporales van creciendo en complejidad, en tanto se encuentran más arriba en el diagrama. Así, por ejemplo, la capa biotemporal es más compleja que la prototemporal en cuanto a las características temporales que implica y los fenómenos que busca explicar, por la cantidad de factores y relaciones que se consideran en ella, (Fraser, 1975).

\section{ATEMPORAL}

La capa atemporal se refiere a los fenómenos en los que no se puede hallar una causalidad explícita que ordene los eventos en un antes y un después. Los fenómenos electromagnéticos son, tal vez, el ejemplo por excelencia. Dado que no es po- sible determinar cuál de las fuerzas en tensión es la que provoca una reacción de los elementos, no podría decirse que un electrón genera el movimiento de otro o que un protón es el responsable del mismo.

De acuerdo con Fraser (1987), "nada en esta capa corresponde a nuestras ideas de pasado-presente-futuro, el paso del tiempo, o temprano-tarde. Nuestra guía para un entendimiento de esta extraña realidad atemporal será la teoría de la relatividad especial de Albert Einstein" (p. 223).

Las situaciones en esta capa de la realidad suceden entre partículas subatómicas a velocidades cercanas a la luz. Este "es un estado de energía donde ninguna de nuestras nociones ordinarias asociadas al tiempo es aplicable" (Fraser, 1987, p. 368). La teoría que Fraser propone para navegar en este nivel de análisis, la teoría de la relatividad especial de Einstein, resulta importante, en el sentido de que muestra las disciplinas que tendrán más interés aquí. Según este autor, "la teoría de la relatividad especial nos dice cosas interesantes acerca de la medición del tiempo en condiciones inusuales, pero no dice qué es el tiempo, la teoría asume que ya sabemos" (Fraser, 1987, p. 233). Podría existir una conceptualización, pero esta teoría no reflexiona respecto a este asunto en particular. 
La manera de denominar esta capa es bastante reveladora. Podría decirse, de algún modo, que no hay tiempo. Las duraciones están por fuera de nuestra percepción y la comparación de eventos como "antes o después" o "mayor o menor duración" están lejos de nuestro alcance, debido a que las mencionadas interacciones electromagnéticas se presentan de manera simultánea para nuestro aparato perceptual $\mathrm{y}$, aunque es posible acceder a ellas, esto surge gracias a la ayuda de experimentos de laboratorio y observaciones realizadas en el espacio (Prigogine, 1980). Esta capa nos ayuda a entender lo comprensivo del modelo de Fraser, pero, considerada de forma individual, podría no intervenir en el análisis de grupos humanos.

\section{PROTOTEMPORAL}

En el nivel prototemporal, los elementos que establecen el ejemplo relevante de comportamiento son las moléculas. Estas uniones de átomos presentan patrones de conducta que se modelan en escenarios probabilísticos, los cuales son sistemas de ecuaciones que consideran al tiempo como una variable reversible. Estos escenarios probabilísticos se diseñan con base en el conocimiento de los estados posibles de la materia (Fraser, 1987).
La denominación de este escenario con el prefijo "proto" trata de dar cuenta del hecho de que aún no es una capa en la que el tiempo se manifieste de manera similar a nuestra experiencia usual en tanto seres humanos (Fraser, 1975). En otras palabras, con la ayuda de instrumentos de precisión, puede identificarse la sucesión de eventos, pero este sigue siendo un escenario aún lejano de la experiencia humana, en tanto individuos o sociedades. De acuerdo con Fraser (1987):

Existe una dificultad para hablar de las formas elementales de la materia. Nuestro lenguaje no tiene palabra para algo que es tan sólido como un melón y también es una onda como una pieza musical. Los llamaré objetos elementales.

Los antropólogos tienen dificultades similares cuando quieren describir culturas diferentes a la propia. Estos deben aprender primero a ver el mundo como es visto por las personas de otras culturas en sus propios términos. Solo entonces, a través del uso de nuevos conceptos, los antropólogos pueden hacer propias las creencias y perspectivas de otra cultura (p. 243).

La problemática de expresión que describe la cita anterior también se presentará en otras capas de análisis, pero en menor medida. Aquí, el 
objeto de estudio central - las moléculas - excluyen el lenguaje de las Ciencias Sociales junto con sus métodos y paradigmas.

En esta capa de la realidad, son protagónicas ciencias como la Química y la Física Cuántica. Estas se apoyan en modelos probabilísticos para intentar determinar el estado futuro de una molécula o identificar su estado anterior a partir del examen del presente (Gell-Mann, 2007). En este punto, se puede identificar cómo se considera al tiempo como un fenómeno externo, lineal y sobre el cual se puede avanzar y retroceder.

Asimismo, es posible identificar probabilidad en la sucesión de eventos, a diferencia de la capa atemporal, en la que los fenómenos electromagnéticos no permiten identificar la partícula subatómica que provoca el movimiento de otra. Como se verá, la siguiente capa de complejidad se acerca aún más a la experiencia humana directa.

\section{EOTEMPORAL}

La tercera capa, la eotemporal, está en el dominio de los cuerpos ina- nimados que podemos percibir a simple vista. Las máquinas son un ejemplo típico. En efecto, la manera de operar de las máquinas y el propósito con el que es diseñada la mayoría de ellas conducen a la ejecución de comportamientos repetitivos durante su existencia. Esta situación es de tiempo reversible, en la que las leyes de la mecánica clásica ${ }^{1}$ se aplican con todo rigor. La capa de eotemporalidad ha sido descrita como pura sucesión, sin una direccionalidad específica (Fraser, 1987).

La pura sucesión es el escenario en donde podemos identificar y comparar la duración de los eventos (Vasco, 2000), sin que nuestra posición temporal con respecto al mismo sea importante. En este escenario, nuestras nociones sobre el pasado, presente y futuro no son relevantes (Fraser, 1975).

Según Fraser (1975), el prefijo "eo" hace referencia a la diosa griega del amanecer. Este autor refiere el movimiento de los astros como otro ejemplo representativo de esta capa. Al hacerlo, invita a considerar los principios de la mecánica clásica y las ideas de Isaac Newton. Estas fueron

1 Entendida como la porción de la Física circunscrita a las leyes de Newton, las cuales son aplicables en escenarios gravitacionales a velocidades pequeñas en comparación con la velocidad de la luz y toman como referencia un objeto inerte (Capra, 2000). La formulación alrededor del movimiento armónico simple es uno de sus ejemplos más representativos. 
esenciales para corregir los modelos astronómicos de la época y lograr predicciones más precisas sobre el movimiento de los astros.

La mecánica clásica funge como una herramienta conceptual para entender el escenario en donde los cuerpos se mueven en el vacío. No se trata solo de un conjunto de ecuaciones conocidas, sino también de la representación de toda una corriente de pensamiento que algunos autores han identificado con el mecanicismo (Etkin \& Schvarstein, 1995).

El mecanicismo, como corriente de pensamiento, implica una visión particular del universo. Esta asigna, a otro tipo de realidades, características de existencia similares a las de las máquinas (Nicol, 1965). Nuestra capacidad de predecir el comportamiento, el estudio de las partes para tratar de alcanzar la comprensión del todo y la necesidad de una posición objetiva son las características más discutidas de esta perspectiva (Morgan, 1997; Múnera, 2007).

El mecanicismo se asocia con el concepto de ciencia reinante a finales del siglo XIX, heredero del cartesianismo y del positivismo, dos corrientes que aportan los supuestos ontológicos y epistemológicos de este paradigma (Ackoff, 2002). Además, se soporta en considerar la posibilidad del conocimiento completo para la predicción y el control por medio de la objetividad y del análisis (García, 2006; Morin, 1988).

La capa eotemporal deja de lado aspectos de nuestra experiencia en tanto seres biológicos, como los ciclos propios de la vida de un organismo y tampoco toma en cuenta nuestra percepción del tiempo o, por ejemplo, el significado cultural que puede tener la interpretación del reloj (Fraser, 1987; Whitrow, 1990).

Las características temporales de esta capa son similares a las de la prototemporal, con la principal diferencia de que aquí el concepto central es causalidad y no probabilidad. En otras palabras, en esta capa, el tiempo es conceptualizado como un parámetro externo, lineal y la diferencia entre pasado, presente y futuro está relacionada con la posición del observador, sin considerar diferencia cualitativa alguna. Esta es una aproximación común y quizás adecuada para campos como la Ingeniería Mecánica o la Electrónica.

\section{BIOTEMPORAL}

En esta capa se estudian los seres vivos. Fraser (1975) llama al tiempo que aquí se considera "el tiempo de la vida", que considera las funciones biológicas y deja para la siguiente 
capa los aspectos cognoscitivos. El tiempo adquiere direccionalidad y los aspectos causales son más importantes.

Esta capa de la realidad incluye círculos que consideran cambios irreversibles en los estados de la materia. Ciclos como la digestión o la respiración son entendidos como partes de una perspectiva de mayor duración dentro de los procesos de crecimiento. Estos procesos, a su vez, implican cambios.

Los cambios en la materia se han estudiado desde diversos puntos de vista. Se brinda una mirada interdisciplinar que combina aspectos de la Física Cuántica y la Biología. Este tipo de aproximación es protagónica en este momento (Fraser, 1987). Desde esta perspectiva, el crecimiento de los organismos es una tendencia de comportamiento y la evolución de las especies es un proceso en el que interviene el caos. Estos procesos de cambio, evolución y crecimiento son momentos de crisis que pueden ser provocados por eventos imprevisibles o caóticos y obligan a los sistemas vivos a modificar sus patrones de comportamiento (Prigogine, 1980; 1989; 1993; 1997).

La idea de biotemporalidad tiene relación con las metáforas biológicas, usadas para ilustrar escenarios de interacción humana desde principios del siglo XX (Fraser, 1987). Las metáforas ayudan a integrar al análisis organizacional elementos propios del mundo biológico, como los ciclos de renovación que se presentan en los sistemas vitales de los mamíferos superiores, entre ellos, la respiración, la circulación sanguínea o la alternancia de descanso y vigilia (Goodwin, 1998). Algunos autores han usado metáforas biológicas como las del organismo, el órgano o el cerebro, para asimilar aspectos propios del funcionamiento de la organización desde diferentes facetas (Morgan, 1997).

Por otra parte, una metáfora también contribuye a entender la complejidad de esta capa de análisis. De acuerdo con Fraser (1987):

Podemos decir que un organismo viviente es una orquesta con trillones de instrumentos que se están interpretando de manera coordinada a cada instante. Esta es una útil y apropiada metáfora, porque nos recuerda la solidaridad orgánica que constituye el proceso vital (p. 128).

La biotemporalidad resalta características como la existencia de múltiples ciclos de duraciones y velocidades diferentes que coexisten en los seres vivos, por ejemplo, el ciclo respiratorio y el ciclo digestivo (Whitrow, 1980; 1990). Si bien hay 
fenómenos similares en los escenarios humanos y sociales, su estudio debe considerar los más variados aspectos relacionados con la conciencia y la cultura.

Las características temporales de la capa biotemporal destacan dos fenómenos importantes: 1) el crecimiento de los seres vivos, y 2) los ciclos de renovación que, por su naturaleza, están en permanente movimiento. El tiempo estudiado en esta capa muestra direccionalidad, en tanto hay evolución y, en consecuencia, no-linealidad, por la presencia de ciclos y retornos de diferentes duraciones. Aquí se permite la construcción de cronología y no de cronometría, es decir, se podrán identificar sucesiones y eventos simultáneos, pero no asignarles magnitudes de duración.

A diferencia de la capa eotemporal, en donde los fenómenos evolutivos o de crecimiento no son tomados en cuenta y en raras ocasiones se analizan procesos de degradación o envejecimiento (Fraser, 1982), estos fenómenos son características centrales de esta capa.

\section{NOOTEMPORAL}

El tiempo de los individuos está en la capa nootemporal o temporalidad noética. En ella, las personas consi- deran su propia experiencia y esta, a la vez, es influida por las consideraciones culturales e históricas conscientes einconscientes(Fraser, 1975).

La experiencia humana abarca múltiples aspectos, entre los que se cuentan la experiencia psicológica de la persona acerca del tiempo, cómo recuerda su pasado y proyecta su futuro, de qué manera la vive en el presente, las variaciones en la percepción de la duración, las consideraciones culturales que constriñen el comportamiento y los controles racionales por parte de los individuos con respecto al tiempo (Boscolo \& Bertrando, 1996).

Al situarse este presente en el contexto de la mente de los seres humanos, se estudia desde los conceptos provenientes de dos ciencias: la Psicología y la Sociología. La primera, por la construcción del concepto de presente en la mente del individuo (Piaget, 1971) y la segunda, porque es el escenario de referencia principal del individuo y de su contexto comunicacional (Maturana, 1997; Zea, 2004).

Los pasados considerados desde cada individuo están marcados por la memoria, la cual se ve influida por la importancia que los actores asignan a cada evento (Fraisse, 1963). Por ejemplo, se recuerda como más 
extenso el lapso pasado en la escuela que aquel transcurrido en casa, porque las consecuencias que se otorgan al paso por los estudios son más significativas que las actividades desarrolladas en casa (Boscolo \& Bertrando, 1996).

Uno de los conceptos empleados en esta capa es la orientación temporal, vinculada con las diferentes tendencias a tomar como principal argumento los eventos en alguna posición temporal, ya sea el pasado, el presente o el futuro. Se pueden observar personas con orientación temporal hacia el pasado, como los adultos mayores, quienes tienden a la añoranza y la repetición de patrones de comportamiento. La orientación temporal hacia el presente es muy poderosa en los niños y adolescentes, quienes son propensos a no examinar las consecuencias de los actos y concentrarse más en la recompensa que en el mismo acto (Piaget, 1970). La tendencia a privilegiar las expectativas y, en general, la orientación temporal hacia el futuro, cuando las decisiones están más concentradas en las consecuencias, es más propia de los jóvenes y adultos (Boscolo \& Bertrando, 1996).

En relación con dicha orientación se halla el concepto de horizonte temporal, explicado como el lapso en el cual se proyecta una entidad. En rea- lidad, las personas tienen diferentes horizontes temporales, relacionados con la experiencia del individuo. Así, cuanta mayor experiencia, mayor será el horizonte temporal (Boscolo \& Bertrando, 1996). Un ejemplo de ello puede ser la manera en la que los ancianos tienden a proyectarse por décadas en el pasado, mientras los jóvenes lo hacen considerando unos cuantos años hacia el futuro.

Las decisiones orientadas al futuro suelen considerar cada vez más factores cuanto más extenso sea el horizonte temporal (Piaget, 1971). La decisión de qué comer al día siguiente, por ejemplo, considera menos factores que una vinculada con el lugar más adecuado para mudarse en los próximos años. Esto no implica una relación lógica rígida, sino que, en ocasiones, los humanos tomamos decisiones más o menos permanentes con criterios o razones perecederas (Mera, 2008).

Las ideas de pasado, presente y futuro son concepciones que anidan en los individuos y se moldean de acuerdo con las circunstancias específicas de cada uno; sin embargo, tienen origen en la cultura de referencia (Beriain, 2008; Valencia García 2007). En esta capa temporal, debe entenderse que, si bien se estudia la temporalidad de una mente humana, esta tiene un marco de 
referencia necesario para que pueda ser explicada (Fraser, 1987).

El pasado de un individuo se relaciona con su cultura, en tanto tenga la capacidad o la prioridad de registrar dos conjuntos de datos: 1) los eventos de la sociedad de referencia que facilitan construir una cronología de hechos, los cuales se pueden tomar como referencia del paso del tiempo, y 2) los hechos personales que permiten rastrear los hitos que marcan y, en ocasiones, medir la duración de la vida de un individuo (Whitrow, 1990). En las sociedades occidentales actuales es posible conocer, con relativa sencillez, cuántos años han transcurrido desde un nacimiento en particular, mas hubo culturas en las que no existía registro de un número, sino de sucesos naturales como un temblor o el desbordamiento de un río (Fagg, 1985; 2003).

El presente mental de los humanos, además de la estimación de la duración, también tiene especial complejidad. Deben atenderse necesidades múltiples que implican diferentes grados de urgencia, satisfacción y placer, así como distintos grados de esfuerzo, lo que, en suma, genera un permanente cambio en los límites del presente mental (Fraser, 1975). En este punto, lo biológico - consciente y no consciente-, lo psicológico y lo social se hacen presentes en la mente, para competir por el esfuerzo y concentración. En la competencia por un esfuerzo presente está la construcción que se ha hecho del futuro, de manera que se perseguirán o no acciones que favorezcan la gratificación futura o la inmediata.

La formación del concepto temporal en cada individuo corresponde a un proceso de asimilación de los códigos culturales desarrollados sobre ese aspecto. La estructura del cerebro que lo posibilita resalta que las personas operan primero con conceptos espaciales concretos y luego los temporales abstractos (Boscolo \& Bertrando, 1996; Piaget, 1971). Los aspectos culturales adquieren relevancia en esta capa como un factor externo que influye en los procesos cognitivos del sujeto. A su vez, la estructura de la mente tiene una jerarquía que retiene la historia de su evolución y suma en la percepción temporal de los actores tanto las urgencias biológicas como los códigos de comportamiento cultural.

Los códigos temporales también inculcan el significado de conceptos como viejo, joven, adulto, rápido y muchos otros que usamos en la cotidianidad. Esto, claro está, en el entendido de que siempre son puestos en contexto, dentro de ciertos límites, en cada cultura (Whitrow, 1990). Así, por ejemplo, las ideas 
de adulto o viejo han ido cambiando para las culturas occidentales a lo largo de la historia (Fagg, 2003).

Esta capa presenta una temporalidad que estudia a los individuos y se interesa, en particular, en su percepción y conceptualización del tiempo. La dimensión temporal está integrada tanto por aspectos de capas anteriores (por ejemplo, la direccionalidad del tiempo propia de la capa biotemporal) como por aspectos propios de la conciencia humana.

El tiempo es asumido en la percepción de los individuos como no lineal. La separación entre pasado, presente y futuro es cualitativa y no solo depende de la posición del observador, como en la capa eotemporal, sino que es parte del objeto de estudio y no un parámetro externo. Esto implica que la edad del sujeto, su formación, su orientación y su horizonte temporal deben ser tenidos en cuenta para la comprensión de la dimensión temporal de las personas (Mera, 2011). Para comprender escenarios de interacción humana, es necesario incluir algunos otros factores como los que se analizan en la siguiente capa temporal.

\section{SOCIOTEMPORAL}

Esta capa del universo requiere la existencia de seres humanos y, por tanto, de la capa nootemporal. Sin embargo, este no es suficiente para explicar los fenómenos globales de la sociedad. Uno de los procesos más notorios en la capa sociotemporal es el desarrollo histórico, por medio del cual las sociedades evolucionan en su relación con el tiempo. La capa sociotemporal es postulada como aquella que abarca la existencia global de los seres humanos e implica el tratamiento social del tiempo así como su concepción colectiva. Fraser (1987) indica:

Desde esta [capa sociotemporal] se trata de una temporalidad - así parece- que está en proceso de creación. Es muy difícil bosquejar sus rasgos con la misma autoridad con que se ha hecho para el tiempo de la luz, partículas sin masa en reposo, macrocuerpos o vida (p. 368).

Este autor considera que su propia posición, en tanto observador, limita las posibilidades de describir los rasgos de comportamiento de la capa sociotemporal. Menciona dos procesos centrales dentro del análisis: 1) la socialización del tiempo, y 2) la evaluación colectiva del tiempo. Fraser (1987) los explica así:

El primero es la coordinación [timing] de las acciones colectivas a través de la sincronización y generación de agendas. Este proceso puede ser llamado socialización del tiempo. 
El segundo proceso es la creación y mantenimiento de un sistema de valores que guíe la conducta de los miembros de una sociedad. Este sistema deriva su autoridad de la historia y los planes del grupo, como son vistos por sus miembros. Este proceso puede ser llamado evaluación colectiva del tiempo (p. 188).

En cuanto al cambio progresivo de los medios de la comunidad, para establecer acuerdos sobre la dimensión temporal, se habla de calendarios y relojes como los medidores esenciales de estos pactos sociales. Esto se relaciona con los valores con los cuales se evalúan (Fagg, 2003). La socialización del tiempo y la evaluación colectiva del mismo están íntimamente relacionadas, pues estos símbolos van condensando más significados en la medida en que una sociedad se desarrolla cada vez más. Por ejemplo, hablar de mayoría de edad acarrea un cúmulo de responsabilidades y derechos que no existía en las sociedades anteriores. Se crean instrumentos que integran cada vez más significados para la socialización del tiempo.

En la evolución de símbolos mediadores en las actividades de socialización del tiempo, como el reloj y los calendarios, es posible ver dos fenómenos: 1) la creciente independencia del tiempo frente a los entornos astrológico y biológico, y 2) la comprobación de un desarrollo desde un punto de vista cultural de los conceptos asociados con el tiempo, como pueden ser la edad, los años y las eras, entre otros (Askin, 1968).

El aspecto de la evaluación colectiva del tiempo parece menos universal que los fenómenos de coordinación alrededor del calendario y el reloj. La evaluación colectiva es parte del sistema de valores, uno de los elementos más complejos de estudio del comportamiento humano y social (Fraser, 1972). Este sistema se relaciona con la historia específica de un grupo social en particular, de manera que es demasiado amplio considerar la cultura occidental en conjunto, como lo hicimos en el caso del calendario. A modo de muestra, no es uniforme el tiempo de espera socialmente correcto en diferentes países occidentales: Inglaterra ha hecho fama de su puntualidad, mientras en países de América Latina, como México y Colombia, parece normal una espera de quince minutos. Los horarios de verano, la hora acostumbrada para el inicio de actividades y la cadencia de las actividades son otros indicadores que diferencian a una cultura de otra.

En esta capa, como en la anterior, el pasado y el futuro son cualitativamente diferentes. Esta es una característica central de lo nootemporal 
y lo sociotemporal. El pasado debe ser construido en el presente, en particular cuando se trata de individuos $\mathrm{y}$, para esto, se requiere memoria. Cuando el pasado pertenece a un grupo específico, es necesario un sistema de registro que construya una memoria colectiva. El futuro, desde la perspectiva de un sujeto, corresponde a una evaluación más causal y parcializada. Para una sociedad, los elementos políticos y de valores históricos envían señales acerca de escenarios posibles (Fraser, 1987).

En esta capa sociotemporal, el presente depende de la sociedad de la que se trata (Fraser, 1987). En la actualidad occidental, los territorios están conectados al instante por tecnologías de información y comunicaciones. Esta situación hace que las culturas y los eventos se combinen y generen una sincronía nunca antes vista. Esta situación produce una gran presión sobre los individuos y los grupos de personas para mantener un ritmo vertiginoso y lograr volúmenes de información que sobrepasen las capacidades humanas (Toffler, 1984; 2000; 2006).

La capa sociotemporal es la más compleja del modelo de Fraser y se nutre de todas las anteriores de manera indirecta y de la nootemporal de modo directo, el cual incluye la conciencia humana. La capa socio- temporal considera un tiempo que deja de ser perceptual, como lo es en parte en la nootemporal, para resaltar los ejercicios de coordinación colectiva, los símbolos necesarios para que surja esa coordinación y el proceso dinámico requerido para la creación de tales símbolos. Esta capa, según propone Fraser (1975; 1987), aporta los elementos para el estudio de la dimensión temporal de un grupo de seres humanos.

\section{LA ORGANIZACIÓN COMO OTRA CAPA DE LA REALIDAD}

Fraser establece que las capas de la realidad así definidas son semiautónomas, lo que significa que tienen características independientes y relaciones con las otras capas, en particular, con las más cercanas. Si se considera la organización como otra capa de la realidad, las más cercanas serían la nootemporal y la sociotemporal. Podríamos denominar "teleotemporal" al nivel de análisis en donde se caracteriza la temporalidad de la organización, si consideramos que el elemento central en la definición y el comportamiento de las organizaciones es el enfoque hacia un objetivo.

En este nivel de análisis, proponemos considerar a la organización, en tanto grupo humano, como un fenómeno paradigmático. Creemos que 
es vital la participación de la teoría de la organización y el concurso de la gestión, la Psicología Social, la Sociología Organizacional y la historia de la empresa como dos aspectos que pueden contribuir a explicar los fenómenos presentes en esta capa temporal.

Tres conceptos han sido identificados como autónomos de la teleotemporalidad. Estos son: feedforward, horizonte temporal y dinámica (Beer, 1979; 1981) (implica ciclos, ritmo y cadencia) y estructura (Mera, 2008). El de feedforward es la tendencia de individuos o grupos a comportarse con base en una expectativa y no en la experiencia (Sanabria, 2003; Foerster, 1991). Esto, en términos de la orientación temporal ilustrada en la capa nootemporal, quiere decir la existencia de una orientación hacia el futuro. La definición de un conjunto de objetivos establece un escenario posible que aún no existe, pero ajusta el comportamiento del actor al mismo (Godet, 1996).

Por su parte, en la capa teleotemporal, el horizonte temporal adquiere un carácter más preciso. Para grupos humanos como las organizaciones, la duración del horizonte que está transcurriendo es más clara y rígida. Las organizaciones trazan planes para períodos determinados y estos impactan en el comportamiento organizacional. Una compañía que planea una innovación anual en su producto estrella se mide y comporta diferente de otra que presta un servicio más o menos estable por décadas, si seguimos los razonamientos de Stacy (1992), por mencionar un ejemplo.

La dinámica se entiende desde varios componentes: el ritmo es la velocidad de sus ciclos principales, mientras la cadenciase refiere al movimiento general de avance. Una compañía que fabrica alfileres tendrá un ritmo veloz, pero una cadencia limitada, mas una consultora empresarial podría tener una relación inversa en lo que atañe a estos dos factores.

El aspecto de los ciclos de una organización se relaciona con la coordinación entre ellos. La mayoría de las organizaciones vinculadas con el mercado están obligadas, por ejemplo, a observar el ciclo fiscal dentro del cual pueden suceder varios ciclos de producción y tal vez solo una determinada porción de un ciclo innovador.

La estructura de una organización tiene una importante correlación con el ambiente en donde se desenvuelve. Los nexos entre los componentes de una organización tienen fuerte correspondencia con su actividad 
principal. Esto la circunscribe a un sector de actividad, a ciertas barreras de entrada y salida y a determinadas velocidades posibles (Eisenhardt \& Brown, 1987; 2002). Generar una innovación anual para una petrolera, por ejemplo, no es una posibilidad; en cambio, se ha vuelto casi una obligación para los productores de telefonía móvil y es una opción presente para otro tipo de organizaciones como los restaurantes.

\section{CONCLUSIONES}

Este artículo propone integrar un nuevo nivel de análisis a un modelo interdisciplinar reconocido y utilizado: el modelo de Fraser (1975; 1987). Este, en su versión clásica, considera seis capas de estudio de la temporalidad. En el trabajo de este autor se hallan elementos útiles para el estudio de la temporalidad de las organizaciones, así como un modelo comprensivo para analizar este fenómeno en escenarios de mayor alcance. Sin embargo, pensamos que los grupos humanos orientados a objetivos presentan suficientes comportamientos autónomos como para dar lugar a un nuevo nivel de estudio, que se ha denominado "teleotemporal" y se ubica en medio de la nootemporalidad y la sociotemporalidad (figura 1).

El comportamiento teleológico de un grupo de humanos exhibe ca- racterísticas de mayor rigidez de estructura que el de los individuos, así como objetivos más medibles y aislables que los de la sociedad. Si bien la propuesta acerca de la teleotemporalidad debe alimentarse de esas capas de la realidad, para que pueda ser estudiada también deben considerarse sus particularidades.

La búsqueda por la comprensión de la temporalidad de la organización no debe entenderse como una propuesta que trata de desplazar a otras, sino que debe integrarse a modelos más comprensivos de la organización como pueden ser los de Stafford Beer (1979; 1981), el de Daniel Katz y Robert Kahn (1989) o la propuesta de Jorge Etkin (1993; 2005; 2007) entre otros, con el fin de hacer un estudio más profundo de este fenómeno tan destacado para el ser humano.

\section{REFERENCIAS}

Ackoff, R. (2002). El paradigma de Ackoff: una administración sistémica. (R. Piña García, trad.) México D. F.: Limusa.

Askin, F. (1968). El problema del tiempo: su interpretación filosófi$c a$. Montevideo: Pueblos Unidos. Battram, A. (2001). Navegar por la complejidad: guía básica sobre la teoría de la complejidad en la empresa y la gestión. Barcelona: Granica. 
Beer, S. (1979). The Heart of Enterprise. Chichester: Wiley.

Beer, S. (1981). Brain of the firm: The managerial cybenetics of organization. Chichester: Wiley.

Beriain, J. (2008). Aceleración y tiranía del presente: la metamorfosis en las estructuras temporales del presente. México D. F.: Antrophos.

Boscolo, L. \& Bertrando, P. (1996). Los tiempos del tiempo: una nueva perspectiva para la consulta y la terapia sistémicas. Barcelona: Paidós.

Capra, F. (2000). El tao de la física (3a ed.). (A. A. Martell Moreno, trad.) Málaga: Sirio.

Eisenhardt, K. \& Brown, S. (1987). Determinación del ritmo en función del tiempo. En Harvard Business Review: La gestión de la incertidumbre (pp. 199-231). Bilbao: Deusto.

Eisenhardt, K. \& Brown, S. (2002). Competir al borde del caos. Madrid: Granica.

Etkin, J. (1993). La doble moral de las organizaciones: los sistemas perversos y la corrupción institucionalizada. Madrid: McGrawHill.

Etkin, J. (2005). Gestión de la complejidad en las organizaciones: la estrategia frente a lo imprevisto y lo impensado. México D. F.: Granica.

Etkin, J. (2007). Capital social y valores en la organización social sustentable: el deber ser, poder hacer y la voluntad creativa. Buenos Aires: Granica.

Etkin, J. \& Schvarstein, L. (1995). Identidad de las organizaciones: invarianza y cambio. Barcelona: Paidós.

Fagg, L. (1985). Two faces of time. Wheaton: The theosophical society.

Fagg, L. (2003). The becoming of time. Londres: Duke University Press.

Foerster, H. V. (1991). Las semillas de la cibernética. Barcelona: Gedisa.

Fraisse, P. (1963). The Psychology of Time. NuevaYork:Harper\& Row.

Fraser, J. T. (1972). Clock making: The most general trade. En The Study of Time II (pp. 339-355). Nueva York: Springer-Verlag.

Fraser, J. T. (1975). Of time, passion, and knowledge: Reflections on the strategy of existence. Nueva Jersey: Princeton University Press.

Fraser, J. T. (1982). The genesis and evolution of time: A critique of interpretation in physics. Amherst: University of Massachusetts Press.

Fraser, J. T. (1987). Time: The familiar stranger. Amherst: University of Massachusetts Press.

García, R. (2000). El conocimiento en construcción: las formulaciones de Jean Piaget a la teoría de sistemas complejos. Barcelona: Gedisa. 
García, R. (2006). Sistemas complejos: concepto, método y fundamentación epistemológica de investigación interdisciplinaria. México D. F.: Gedisa.

Gell-Mann, M. (2007). El quark y el jaguar: aventuras en lo simple y lo complejo (6a ed.). (A. García \& R. Pastor, trads.) Barcelona: Tusquets.

Godet, M. (1996). De la anticipación a la acción: manual de prospectiva y estrategia. Bogotá: Alfaomega.

Goodwin, B. (1998). Las manchas del leopardo: la evolución de la complejidad. (A. García, trad.) Barcelona: Tusquets.

Hawking, S. (1995). Breve historia del tiempo. Bogotá: Planeta.

Katz, D. \& Kahn, R. (1989). Psicología Social de las organizaciones. México D. F.: Trillas.

Maturana, H. R. (1997). El sentido de lo humano. Santiago: Dolmen.

Mera, C. (2008). Hacia un concepto de tiempo para la organización. (Tesis de maestría inédita). Universidad Nacional de Colombia, Bogotá, Colombia.

Mera, C. (2011). Características del tiempo en la organización. Conferencia presentada en el XVI Congreso Internacional de Contaduría Administración e Informática, México D. F., Universidad Nacional Autónoma de México.

Montoya Restrepo, I. \& Montoya Restrepo, L. A. (2009). Algu- nas concepciones del tiempo en Ciencias Sociales e implicaciones para la investigación en dirección estratégica. Revista Facultad de Ciencias Económicas: Investigación y reflexión, XVII(1), 57-79.

Morgan, G. (1997). Images of organization. Londres: SAGE.

Morin, E. (1988). El conocimiento del conocimiento. Madrid: Cátedra.

Múnera, P. (2007). La idea de organización. Medellín: Comunicación S. A.

Nicol, E. (1965). Principios de la ciencia. México D. F.: Fondo de Cultura Económica.

Piaget, J. (1970). The child's conception of time. Nueva York: Basic Books.

Piaget, J. (1971). La epistemología del tiempo. Buenos Aires: El Ateneo.

Prigogine, I. (1980). From being to becoming: Time and complexity in the physical sciences. San Francisco: Freeman.

Prigogine, I. (1989). Exploring the complexity: An introduction. Múnich: Piper Gmbh \& Co.

Prigogine, I. (1993). ¿Tan solo una ilusión? Barcelona: Tusquets.

Prigogine, I. (1997). El nacimiento del tiempo. Madrid: Taurus.

Sanabria, M. (2003). La temporalidad de la decisión, la crisis y la construcción de la realidad organizacional. Innovar (22), 73-84. 
Stacey, R. (1992). La estrategia de los 90. Bogotá: Legis.

Toffler, A. (1984). El "shock" del futuro. Barcelona: Plaza \& Janés.

Toffler, A. (2000). La tercera ola. Barcelona: Plaza \& Janés.

Toffler, A. (2006). La revolución de la riqueza. Barcelona: Plaza \& Janés.

Valencia García, G. (2007). Entre cronos y kairós. Las formas del tiempo sociohistórico. México D. F.: Anthropos.

Vasco, C. (2000). El tiempo en la teoría general de procesos. En J. Lopera (ed.), El problema del tiempo (pp. 215-240). Medellín: Universidad Nacional de Colombia, Facultad de Ciencias Humanas y Económicas.

Whitrow, G. J. (1980). The Natural Philosophy of Time (2a ed.). Oxford: Clarendon Press.

Whitrow, G. J. (1990). El tiempo en la historia: la evolución de nuestro sentido del tiempo y la perspectiva temporal. Barcelona: Crítica.

Zea, L. F. (2004). La organización como tejido conversacional. Medellín: Fondo Editorial Universidad Eafit. 